\title{
Memories of Shoshichi Kobayashi
}

\section{by $\mathrm{H}$. Blaine Lawson}

I first became personally acquainted with Shoshichi when I moved to Berkeley as a junior faculty member in 1969. Prior to that I knew him principally as the one of the two authors of the elegant and powerful treatise: Foundations of Differential Geometry, which was published six years before. That book had become, for me, the place to go whenever I wanted a clear vision of something in geometry. On the other hand its exposition, while elegant, was terse and unforgiving. As a result, I imagined Kobayashi might himself be an imposing and formidable personality. Nothing could have been further from the truth. I found Shoshichi to be warm and inclusive. He was engaging and had great charm. I remember well the big smile and the light in his eyes. He was one of the people that made the environment for young geometers at Berkeley so wonderful.

In the late sixties Berkeley had collected an amazing group of people in geometry, both junior and senior. It was a period of high productivity in the field. At the core of that group were Shiing-Shen Chern and Shoshichi Kobayashi. They set a tone that made mathematics alive, personal and exciting.

Mathematically of course Shoshichi Kobayashi was a giant. One of his achievements, which I have always admired, was his construction of the Kobayashi metric on complex manifolds. This intrinsic distance has the important property that it decreases (is non-increasing) under holomorphic mappings, and it agrees with standard complete metrics on certain bounded domains. On the other hand it is identically zero on the complex numbers $C$, and therefore, since the distance is intrinsic, if two points in a complex manifold $X$ lie in the image of a holomorphic map $C \rightarrow X$, the Kobayashi distance between them is zero. Thus, manifolds for which the Kobayashi distance is a true metric (these are called Kobayashi hyperbolic manifolds), can never receive such mappings unless they are constant. So his construction opened a vast geometric perspective on Picard phenomena.

This work was completely different from most of Shoshichi's previous research, and it engendered decades of activity in complex analysis, algebraic geometry and number theory. It is now one of the fundamental tools/concepts in the field.

To return to my initial point: even putting all mathematics aside, Shoshichi Kobayashi was a great man. As many have testified, he was a very caring person. In return he was someone for whom we all felt a great deal of affection. 